\title{
Enhancement of Fear Extinction with Deep Brain Stimulation: Evidence for Medial Orbitofrontal Involvement
}

\author{
Jose Rodriguez-Romaguera ${ }^{1,2}$, Fabricio H Do-Monte ${ }^{1,2}$, Yoko Tanimura ${ }^{3}$, Gregory J Quirk*,1,2 and \\ Suzanne N Haber $^{3}$ \\ 'Department of Psychiatry, University of Puerto Rico — School of Medicine, San Juan, PR, Puerto Rico; '2 Department of Anatomy and Neurobiology, \\ University of Puerto Rico — School of Medicine, San Juan, PR, Puerto Rico; ${ }^{3}$ Department of Pharmacology and Physiology, University of Rochester \\ School of Medicine, Rochester, NY, USA
}

\begin{abstract}
Deep brain stimulation (DBS) of the ventral capsule/ventral striatum (VCNS) reduces anxiety, fear, and compulsive symptoms in patients suffering from refractory obsessive-compulsive disorder. In a rodent model, DBS-like high-frequency stimulation of VS can either enhance or impair extinction of conditioned fear, depending on the location of electrodes within VS (dorsal vs ventral). As striatal DBS activates fibers descending from the cortex, we reasoned that the differing effects on extinction may reflect differences in cortical sources of fibers passing through dorsal-VS and ventral-VS. In agreement with prior anatomical studies, we found that infralimbic (IL) and anterior insular (Al) cortices project densely through ventral-VS, the site where DBS impaired extinction. Contrary to IL and Al, we found that medial orbitofrontal cortex (mOFC) projects densely through dorsal-VS, the site where DBS enhanced extinction. Furthermore, pharmacological inactivation of mOFC reduced conditioned fear and DBS of dorsal-VS-induced plasticity ( $\mathrm{pERK}$ ) in $\mathrm{mOFC}$ neurons. Our results support the idea that VS DBS modulates fear extinction by stimulating specific fibers descending from $\mathrm{mOFC}$ and prefrontal cortices.

Neuropsychopharmacology (20I5) 40, I726-1733; do::I0.1038/npp.20 I5.20; published online I I February 2015
\end{abstract}

\section{INTRODUCTION}

Deep brain stimulation (DBS) of the ventral capsule/ventral striatum (VC/VS) reduces fear and anxiety symptoms in patients suffering from refractory obsessive compulsive disorder (OCD; Denys et al, 2010; Greenberg et al, 2010a), but little is known about the mechanisms. Previous studies have proposed that the primary effects of DBS are on cortical fiber bundles passing through the striatum (see Greenberg et al, 2010b for review). Furthermore, OCD patients show deficient extinction of conditioned fear, which depends on specific areas of the prefrontal cortex (Milad et al, 2013). Using a rat model, we previously found that high-frequency DBS-like electrical stimulation of the dorsal-VS (just above the anterior commissure) enhanced fear extinction, whereas stimulation of the ventral-VS impaired fear extinction (Rodriguez-Romaguera et al, 2012). Interestingly, these striatal sub-regions did not follow core/shell compartmentalization. Furthermore, DBS effects were not observed with pharmacological inactivation of dorsal-VS or ventral-VS (Rodriguez-Romaguera et al,

*Correspondence: Dr GJ Quirk, Department of Psychiatry, University of Puerto Rico_-School of Medicine, PO Box 365067, San Juan, PR 00936-5067, Puerto Rico, Tel: +787999 3058, Fax: +787999 3057, E-mail: gregoryjquirk@gmail.com

Received 8 September 2014; revised 9 January 2015; accepted II January 2015; accepted article preview online 20 January 2015
2012), suggesting that DBS modulates different sets of cortical fibers passing through these VS areas. We therefore sought to determine the cortical sources of fibers passing through the dorsal-VS and ventral-VS sub-regions.

The infralimbic(IL) cortex and prelimbic (PL) cortex project through VS (Berendse et al, 1992; Mailly et al, 2013; McGeorge and Faull, 1989; Sesack et al, 1989; St Onge et al, 2012; Vertes, 2004) and could potentially mediate DBS effects on extinction. It is well established that inhibition of IL impairs fear extinction (Burgos-Robles et al, 2007; Laurent and Westbrook, 2009; Quirk et al, 2000; SierraMercado et al, 2011), whereas stimulation of IL enhances extinction (Kim et al, 2010; Maroun et al, 2012; Milad and Quirk, 2002; Milad et al, 2004). In contrast, inhibition of PL reduces fear expression (Corcoran and Quirk, 2007; Laurent and Westbrook, 2009; Sierra-Mercado et al, 2011), whereas PL stimulation increases fear expression and impairs extinction (Vidal-Gonzalez et al, 2006).

Other areas projecting through the VS include the orbitofrontal cortex (OFC) and the anterior insular (AI) cortex (Gabbott et al, 2005; Hoover and Vertes, 2007, 2011; Mailly et al, 2013; McGeorge and Faull, 1989; Schilman et al, 2008), but their role in fear extinction has not been studied. Furthermore, the projection patterns of these areas through dorsal-VS and ventral-VS are not clearly defined. We therefore injected the retrograde tracer wheat germ agglutinin (WGA) into the dorsal-VS or ventral-VS, to assess retrograde labeling in the cortex. Cortical areas 
projecting predominantly through the dorsal-VS site (in which DBS enhanced fear extinction) were further studied using pharmacological inactivation and immunocytochemistry.

\section{MATERIALS AND METHODS}

\section{Subjects}

Fifty five male Sprague-Dawley rats $(\sim 325 \mathrm{~g}$; Harlan Laboratories) were housed and handled as previously described (Quirk et al, 2000). Rats used in the fear conditioning experiment were fed standard rat chow in a restricted manner $(18 \mathrm{~g} /$ day), to facilitate pressing a bar for food on a variable interval schedule of reinforcement (VI-60) and to maintain a constant behavioral baseline against which freezing could be reliably measured. All procedures were approved by the Institutional Animal Care and Use Committee of the University of Puerto Rico-School of Medicine in compliance with the National Institutes of Health guidelines for the care and use of laboratory animals.

\section{Surgery and Drug Infusion}

Rats were initially anesthetized with isofluorane inhalant gas $(5 \%)$ in an induction chamber and positioned in a stereotaxic frame. Isofluorane (2\%-3\%) was delivered through a face mask for anesthesia maintenance. For our neuroanatomical study, unilateral infusions of WGA were aimed at the dorsal-VS $(-6.5 \mathrm{~mm} \mathrm{DV}, \pm 2.0 \mathrm{~mm} \mathrm{ML}$, and $+1.2 \mathrm{~mm} \mathrm{AP})$ or ventral-VS $(-8.0 \mathrm{~mm} \mathrm{DV}, \pm 2.0 \mathrm{~mm}$ ML, and $+1.2 \mathrm{~mm}$ AP; Paxinos and Watson, 1998). A small volume of $20 \mathrm{nl}$ was used to restrict the tracer from spreading into adjacent sites. Using a $0.5-\mu \mathrm{l}$ Hamilton syringe, we infused WGA at a rate of $1 \mathrm{nl} / \mathrm{min}$ during a 20 min period. After infusion, the syringe was left in place for an additional $20 \mathrm{~min}$ to allow for diffusion and avoid backflow through the syringe track. The incision was then sutured using wax-coated braided silk. After surgery, a topical triple antibiotic was applied around the surgery wound and an analgesic (Ketoprofen, $5 \mathrm{mg} / \mathrm{kg}$ ) was injected intramuscularly. Animals were killed 7 days after infusions, to allow for adequate tracer transport.

For our inactivation experiment, fluorescent muscimol (MUS, $0.2 \mu \mathrm{l}, 0.11 \mathrm{nmol}$; BODIPY TMR-X Conjugate; SigmaAldrich, St Louis, MO) was infused to enhance GABA-A receptor activity, thereby temporally inactivating the region. A similar surgical procedure as above was used, except that rats were chronically implanted with 26-gauge bilateral guide cannulas (Plastics One, Roanoke, VA) between medial OFC (MO) and the rostral part of the ventrolateral OFC (rVLO): $+4.68 \mathrm{~mm} \mathrm{AP,} \pm 0.60 \mathrm{~mm} \mathrm{ML},+4.00 \mathrm{~mm} \mathrm{DV}$; Paxinos and Watson, 1998), in order to target the area that resembles the projection to dorsal-VS (Figure 2). Acrylic cement was used to affix cannulas to the skull. Stainless steel obturators (33 gauge) were inserted into the guide cannulas to avoid obstructions until infusions were made. Injector tips extended $1.0 \mathrm{~mm}$ beyond the guide cannula. Rats were allowed 7 days to recover from surgery before behavioral testing. On the day before the behavioral experiment, obturators were removed, injectors were inserted into the cannulas without infusion, and rats were acclimated for handling. On the day of infusion, $0.2 \mu \mathrm{l}$ of MUS or saline (SAL, vehicle) was infused at a rate of $0.2 \mu \mathrm{l} / \mathrm{min}$. After infusion, injectors were left in place for $2 \mathrm{~min}$, to allow the drug to diffuse. After behavioral experiments, rats were perfused and placements were verified for correct placement as previously described (RodriguezRomaguera et al, 2012). Nine rats with placements and MUS spread outside of mOFC were eliminated.

\section{Immunocytochemistry}

For our neuroanatomical study, sections (50 $\mu \mathrm{m}$ thickness, $200 \mu \mathrm{m}$ apart) were initially treated with $10 \%$ methanol and $3 \% \mathrm{H}_{2} \mathrm{O}_{2}$ in $0.1 \mathrm{M} \mathrm{PB}$, to inhibit endogenous peroxidase, and rinsed extensively in PBS with Triton X-100 (PB-TX; Sigma, St Louis, MO). Preincubation occurred in PB-TX with $10 \%$ normal goat serum (NGS) for $30 \mathrm{~min}$ and incubation in primary anti-WGA (1:50 000; Sigma-Aldrich) in PBS-TX with $10 \%$ NGS for four nights at $4{ }^{\circ} \mathrm{C}$. The tissue was then treated with biotinylated secondary antibody $(1: 200$; Vector Laboratories, Burlingame, CA) and incubated with avidin-biotin complex solution (Vectastain $\mathrm{ABC}$ kit; Vector Laboratories). Standard DAB procedures were used to react the tissue and visualization was optimized by using cobalt chloride and nickel ammonium sulfate. Sections were mounted onto gel-coated slides, dehydrated, defatted in xylene, and coverslipped with mounting medium (Permount, Fisher Scientific, Fair Lawn, NJ). To visualize the cytoarchitectonic subregions of cortical area, adjacent sections were reacted with neuronal marker NeuN. Sections were incubated with primary anti-NeuN antibody $(1: 1000$; Chemicon, Rosemont, IL) overnight at room temperature and treated with the same procedures described above.

For our pERK immunocytochemistry experiment, alternate sections were initially blocked in a solution of $2 \%$ NGS (Vector Laboratories, USA) plus $0.3 \%$ triton (Triton X-100, Sigma-Aldrich) in $0.12 \mathrm{M}$ potassium buffer SAL for $1 \mathrm{~h}$, as previously described (Rodriguez-Romaguera et al, 2012). The sections were incubated overnight at room temperature with anti-pERK serum raised in rabbit $(1: 100$; p-44/ 42MAPK, 4370L; Invitrogen, Carlsbad, CA) and treated with the same procedures described above.

For our neuroanatomical study, the tissue from a total of 24 WGA-injected animals was examined. Striatal injections with either contamination or weak labeling were eliminated from the analysis. Contamination refers to injections in which the tracer was not limited to the dorsal-VS or ventral-VS regions, but had leaked into the adjacent striatal regions. Weak labeling refers to poor uptake of the tracer as indicated by poor labeling in the midbrain. A total of four animals (two in dorsal-VS and two in ventral-VS) had injection sites that were confined to the dorsal or ventral region with adequate uptake. The cortex was divided into specific regions based on the rat atlas (Paxinos and Watson, 1998) and labeled cells were plotted with stereological analysis (see below). Cortical areas were defined according to cytoarchitechtonic boundaries in adjacent sections, as indicated by NeuN labeling (Paxinos and Watson, 1998). We analyzed the number of labeled cells in the medial (mPFC), OFC, and AI prefrontal cortices. The mPFC was subdivided into cingulate (Cg1), PL, and IL cortices, the 
OFC into MO and VLO OFCs, and the AI into ventral (AIv) and dorsal (AId) AI cortices.

The number of cells labeled with WGA was quantified by using an unbiased stereology method (Stereoinvestigator Software, Micro Bright Field, Williston, VT), with a modified Nikon microscope (Nikon E800) under dark field illumination. Contours were drawn for each cortical area leaving a small transitional space $(\sim 200 \mu \mathrm{m})$ between areas. A fractionator probe was used to estimate the number of labeled cells with systematic random sampling. Quantification of cells was performed at $\times 20$ magnification in $100 \mu \mathrm{m}^{2}$ counting frames, which constituted $64 \%$ of the reference space. We used the PL cortex to confirm that stereology estimates were accurate by comparing with manual counts of the entire structure. For each cortical region, the fraction of cells (\% of total cells counted) was used to analyze the distribution of cortical cell labeling. The fraction of cells between each injection site was compared for dorsal-VS and ventral-VS regions.

For our pERK immunocytochemistry experiment, images were generated for mOFC and the number of pERK immunoreactive neurons in each brain hemisphere was quantified as previously described (Rodriguez-Romaguera et al, 2012). The density of pERK-positive neurons was calculated by dividing the number of positive neurons by the total area counted.

\section{Behavior}

Rats underwent bar-press training, auditory fear conditioning, and extinction in standard operant chambers (Coulbourn Instruments, Whitehall, PA) inside soundattenuating boxes (Med Associates, St Albans, VT). Further details regarding the apparatus have been previously described (Quirk et al, 2000). On day 1, rats were presented with five non-reinforced tones $(4 \mathrm{kHz}, 75 \mathrm{~dB}, 30 \mathrm{~s}$; habituation) followed by seven tones that co-terminated with footshocks ( $0.5 \mathrm{~s}, 0.56 \mathrm{~mA}$; conditioning). On day 2, rats were infused with either SAL or MUS $30 \mathrm{~min}$ before being presented with 15 tones in the absence of footshocks (extinction). On day 3, rats were presented with eight tones in the absence of footshocks (extinction test). The first trial of habituation, the last trial of conditioning, and the last trial of extinction were eliminated from analysis to make two trial blocks (according to Rodriguez-Romaguera et al, 2012). During all phases of the experiment, the intertone interval was variable $(\sim 3 \mathrm{~min})$ and food was available on a variable interval schedule (VI-60).

\section{Deep Brain Stimulation}

DBS was delivered through concentric bipolar electrodes (NEX-100; Rhodes Medical Instruments, Tujunga, CA) with contacts measuring $0.5 \mathrm{~mm}$ in length and separated by $0.5 \mathrm{~mm}$ as previously described (Rodriguez-Romaguera et al, 2012). Stimulation was monophasic, with the deeper contact as negative. We used DBS parameters similar to those used in humans $(100 \mu \mathrm{A}, 0.1-\mathrm{ms}$ pulse duration, $130 \mathrm{~Hz}$ ), which were also used in previous rat models studying DBS-like stimulation (Do-Monte et al, 2013; McCracken and Grace, 2007; Rodriguez-Romaguera et al, 2012). DBS was generated with an S88X stimulator (Grass
Instruments, Warwick, RI) and a constant-current unit (SIC-C Isolation Unit; Grass Instruments).

\section{Data Collection and Analysis}

Behavior was recorded with digital video cameras (Micro Video Products; Bobcaygeon, Ontario, Canada) and freezing was quantified from digitized video images using commercially available software (Freezescan; Clever Systems, Reston, VA). The amount of time rats spent freezing to the tone was expressed as a percentage of the tone presentation. Statistical significance was determined with Student's two-tailed $t$ tests, one-way ANOVA, or repeatedmeasures ANOVA, followed by Tukey post-hoc analysis, when appropriate (STATISTICA; Statsoft, Tulsa, OK).

\section{RESULTS}

Figure 1a shows findings from our previous study (Rodriguez-Romaguera et al, 2012), in which DBS of ventral-VS impaired fear extinction, and DBS of dorsalVS enhanced fear extinction. To identify the cortical areas projecting through these different VS subregions, we injected the retrograde tracer WGA into either dorsal-VS or ventral-VS, and used stereological techniques to count the number of retrogradely labeled cells in different cortical areas (Figure $1 \mathrm{~b}$ and $\mathrm{c}$ ).

\section{Ventral-VS Receives Projections from PL, IL, and AI}

Figure $2 \mathrm{a}$ and $\mathrm{b}$ show drawings of retrogradely labeled neurons resulting from WGA injections into dorsal-VS or ventral-VS. Group data revealed that there were no significant differences between the two striatal sites in the number of labeled cells in PL (dorsal-VS: 46\%, ventral-VS: $45 \%, t_{(3)}=0.59, p=0.62$; Figure $2 \mathrm{c}$ ), Cg1 (dorsal-VS: $4 \%$, ventral-VS: $\left.3 \%, t_{(3)}=0.96, p=0.44\right)$, or caudal VLO (cVLO; dorsal-VS: $15 \%$, ventral-VS: $\left.16 \%, t_{(3)}=-0.02, p=0.98\right)$. Regarding PL, the ventral-VS projections originated largely from middle and deep layers, but not superficial layers. In contrast to $\mathrm{PL}, \mathrm{Cg} 1$, and $\mathrm{cVLO}$, there were significant differences between striatal sites in the number of labeled cells in the IL and AI (AId + AIv) cortices. Specifically, ventral-VS injections yielded significantly more IL and AI labeling than dorsal-VS injections (IL = dorsal-VS: $3 \%$, ventral-VS: $24 \%, t_{(3)}=-4.90, p=0.04 ; \mathrm{AI}=$ dorsal-VS: $4 \%$, ventral-VS: $17 \%, t_{(3)}=-5.66, p=0.03$; Figure $2 \mathrm{~b}$ and $c$ ). This suggests that modulation of IL and AI may contribute to the impairment of extinction by DBS of ventral-VS, perhaps through DBS-induced inhibition of IL, as previously proposed for lateral OFC (McCracken and Grace, 2007).

\section{Dorsal-VS Receives Projections from PL and mOFC}

In contrast to ventral-VS, projections from PL to dorsal-VS originated largely from middle and superficial layers, but not deep layers. Furthermore, dorsal-VS tracer yielded significantly more retrograde labeling in the $\mathrm{mOFC}(\mathrm{MO}+$ rVLO), compared with ventral-VS (mOFC; dorsal-VS: 43\%, ventral-VS: $11 \%, t_{(3)}=10.99, p=0.008$; Figure $2 \mathrm{a}$ and $\mathrm{c}$ ). Thus, DBS enhancement of extinction observed with the 
a

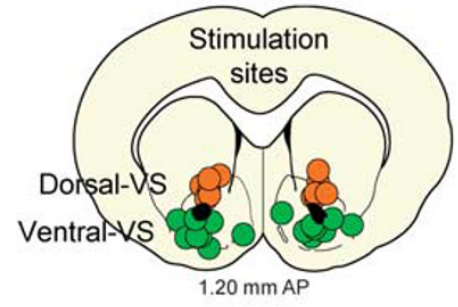

(from Rodriguez-Romaguera et al., 2012)

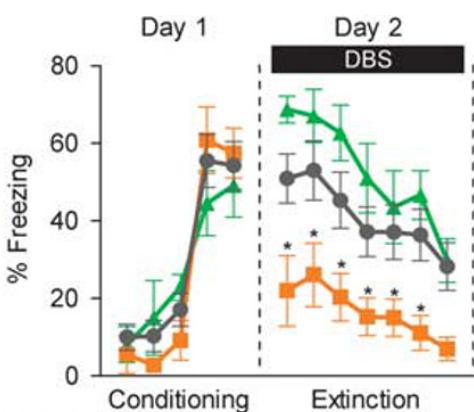

Day 3 b
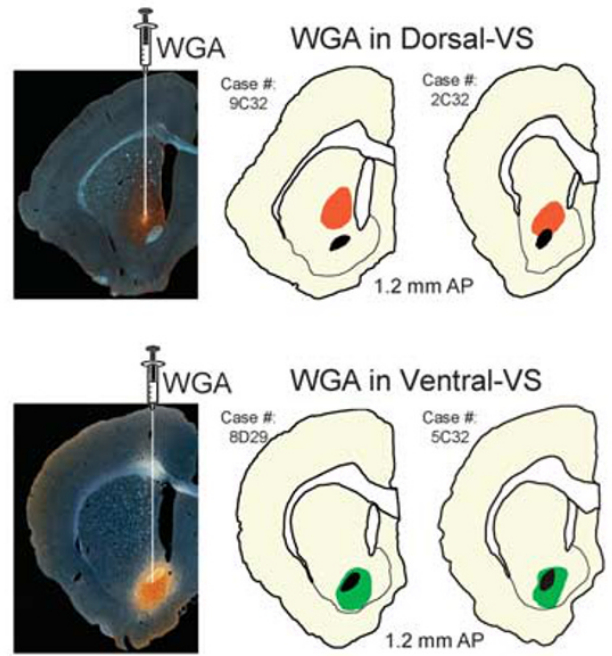

C

Retrograde labeling (non-biased stereology)

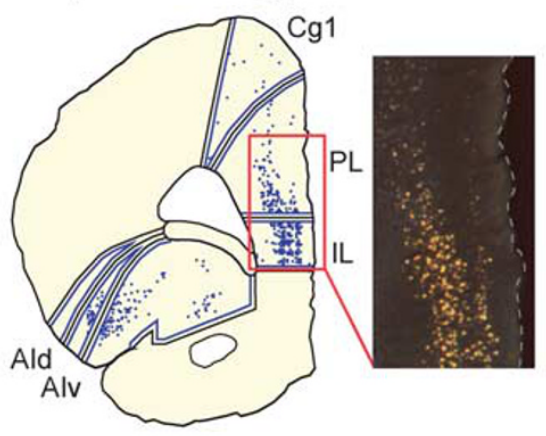

Figure I Stereological analysis of cortical neurons projecting through dorsal- and ventral-ventral striatum (VS). (a) Left: deep brain stimulation (DBS) electrode placements in VS. Circle diameter indicates the estimated spread of current from electrode tip. DBS of dorsal-VS was applied dorsal to anterior commissure (orange circles) and DBS of ventral-VS was applied ventral to anterior commissure (green circles). Right: freezing plots for Sham ( $n=16$ ), dorsal-VS DBS $(n=6)$, and ventral-VS DBS $(n=8)$ groups. Dorsal-VS DBS reduced freezing on day 2 and facilitated extinction recall on day 3. Ventral-VS DBS had the opposite effects. Data are shown in blocks of two trials as mean $\pm \mathrm{SEM}$. $* p<0.05$. Adapted from Figure Ib and c of Rodriguez-Romaguera et al, 2012. (b) Left: representative micrographs showing the spread of wheat germ agglutinin (WGA) into dorsal-VS (top) or ventral-VS (bottom). Right: Injection sites and spread of all cases used in Figure 2c. (c) Illustration of the cortical maps generated using non-biased stereology techniques. Sample obtained from a rat with a WGA injection into ventral-VS.

dorsal-VS site could be due to DBS-induced inhibition of mOFC (McCracken and Grace, 2007), if mOFC normally opposes extinction. Prior studies, however, have not examined the role of $\mathrm{mOFC}$ in fear expression or fear extinction.

\section{Pharmacological Inactivation of mOFC Reduces Fear Expression}

To determine the role of $\mathrm{mOFC}$ in fear expression and extinction, we infused the fluorescently labeled GABA-A agonist MUS into $\mathrm{mOFC}$ just before extinction training (on Day 2). We included only rats showing spread of MUS to $\mathrm{MO}$ and $\mathrm{rVLO}$ subregions of mOFC (Figure 3a). Inactivation of $\mathrm{mOFC}$ significantly reduced freezing from the start of the extinction session (Figure 3b). Repeated-measures ANOVA revealed a main effect of group $\left(\mathrm{F}_{(1,12)}=12.46, p=0.004\right)$. Post-hoc comparisons confirmed that MUS-infused rats showed significantly less freezing than controls in trial blocks 1, 2, 3, 5 and 7 (all $p$ 's $<0.05$ ), suggesting that mOFC activity acts to augment fear expression. The following day, both SAL and MUS rats showed significantly less freezing than the previous day $\left(t_{(7)}=2.47, p=0.04\right)$, demonstrating extinction retrieval (Figure $3 b)$. There was no difference, however, between MUS and SAL groups in extinction retrieval $\left(\mathrm{F}_{(1,12)}=0.04, p=0.84\right)$, indicating that $\mathrm{mOFC}$ is not a site of extinction memory.

\section{DBS of Dorsal-VS Induces Plasticity in mOFC}

Our mOFC MUS finding is consistent with the idea that DBS-induced inhibition of mOFC could account for reduced freezing observed when DBS is on (see Figure 1a), but what accounts for reduced freezing the following day when DBS is off? One possibility is that DBS (but not extinction itself) induces plasticity in inhibitory circuits within mOFC (Gradinaru et al, 2009; McCracken and Grace, 2007), which could account for the long-lasting reduction in freezing. To investigate whether DBS alone induces plasticity in mOFC, we measured the expression of the neuronal plasticity marker pERK (Sweatt, 2004). Naive rats were given $3 \mathrm{~h}$ of DBS or sham stimulation in either dorsal-VS or ventral-VS (same as Figure 1a), and were killed immediately after. We observed that DBS of dorsal-VS (but not ventral-VS) triggered pERK labeling in mOFC neurons. One-way ANOVA revealed a significant effect of treatment in the 

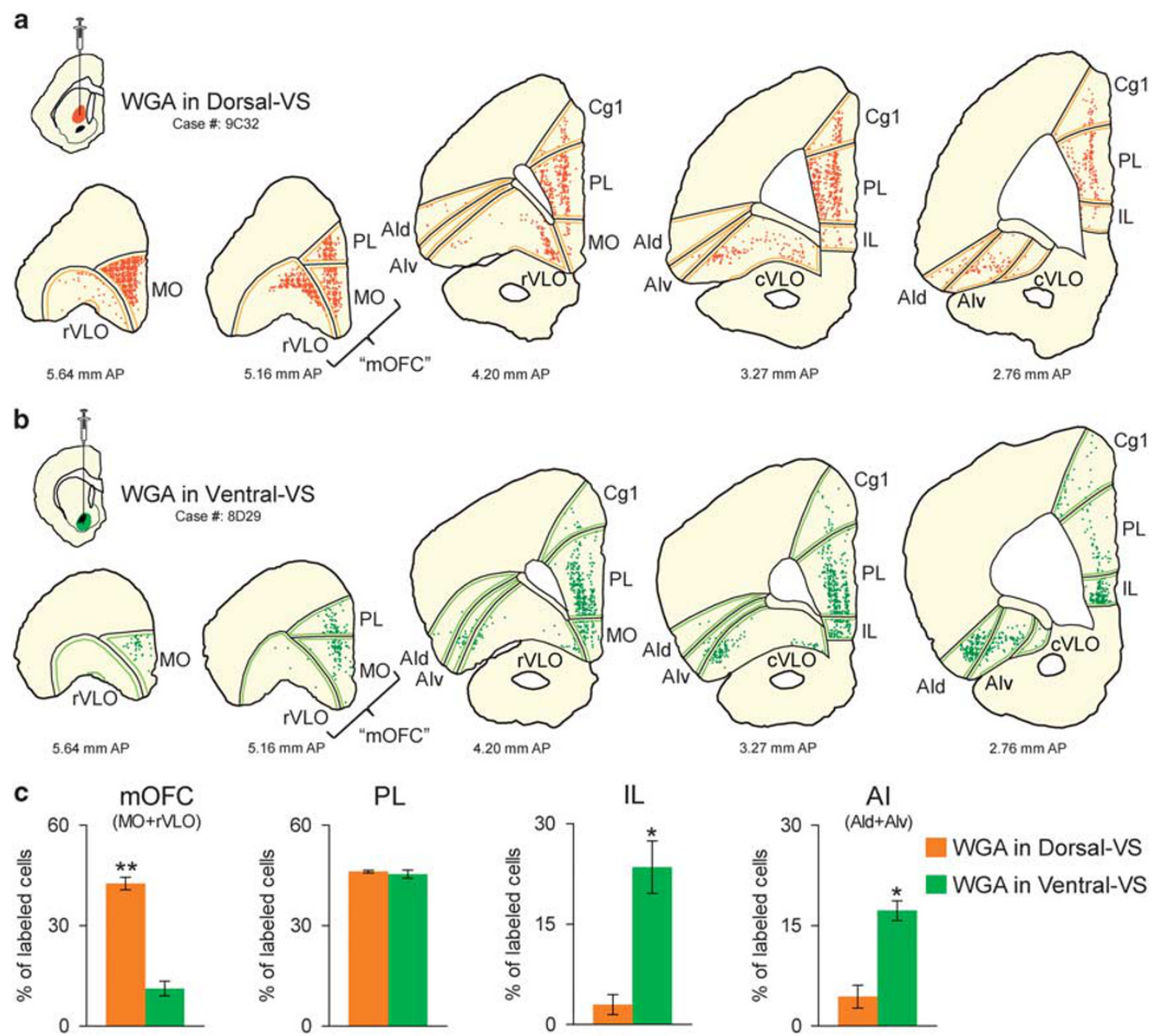

Figure 2 Medial orbitofrontal cortex (mOFC) projects through dorsal-ventral striatum (VS), whereas infralimbic (IL) and anterior insular (AI) cortices project through ventral-VS. (a) Stereological maps of cortical neurons that project through dorsal-VS (orange dots) at various anterior-posterior (AP) levels from bregma. Top left inset: micrographs showing the spread of wheat germ agglutinin (WGA) into dorsal-VS that generated the stereological maps. The boundary of the cortical area counted is defined using orange lines. (b) Stereological maps of cortical neurons that project through ventral-VS (green dots) at various AP levels from bregma. Top left inset: Micrographs showing the spread of WGA into ventral-VS that generated the stereological maps. The boundary of the cortical area counted is defined using green lines. (c) Comparison of fraction of cells (\% of total cells counted) retrogradely labeled by WGA infusions into either dorsal-VS or ventral-VS $(n=4)$. Data presented as mean \pm SEM. $* p<0.05, * * p<0.01$. Ald, dorsal portion of anterior insular; Alv, ventral portion of anterior insular; $\mathrm{CgI}$, cingulate area I; cVLO, caudal portion of ventrolateral orbitofrontal; $\mathrm{MO}$, medial orbitofrontal; $\mathrm{PL}$, prelimbic; rVLO, rostral portion of ventrolateral orbitofrontal.

number of pERK-labeled neurons within $\operatorname{mOFC}\left(\mathrm{F}_{(2,31)}=\right.$ 15.90, $p<0.001$; Figure 4). Post-hoc tests confirmed that DBS of dorsal-VS significantly increased the number of pERK-labeled neurons compared with sham-operated controls $(p<0.001)$. Further studies are needed to discriminate between plasticity in excitatory $v s$ inhibitory networks within mOFC.

\section{DISCUSSION}

We investigated cortical projections through the dorsal and ventral subregions of ventral striatum, to better understand the effects of DBS on extinction of conditioned fear (Rodriguez-Romaguera et al, 2012). We found that dorsal and ventral subregions of VS receive different sets of inputs from $\mathrm{mPFC}$ and OFC. The dorsal-VS receives strong projections from $\mathrm{mOFC}$, whereas the ventral-VS receives strong projections from IL and AI. Thus, enhancement of fear extinction with DBS of dorsal-VS could be due to modulation of mOFC fibers, whereas impairment of fear extinction with DBS of ventral-VS could be due to modulation of IL/AI fibers.

Pharmacological inactivation of mOFC reduced freezing, demonstrating that mOFC is part of fear expression circuitry. mOFC could drive fear through its projections to the basolateral nucleus of the amygdala or PL (Hoover and Vertes, 2011). Therefore, one could speculate that DBS of dorsal-VS inhibits the mOFC. McCracken and Grace (2007) have shown that DBS-like stimulation of dorsal-VS reduces OFC firing rate, and suggest that this occurs via activation of recurrent collaterals projecting onto inhibitory interneurons in OFC. Thus, reduced freezing in the presence of DBS could be due to inhibition of mOFC. Projections from deep layers of PL through dorsal-VS may also be 
a
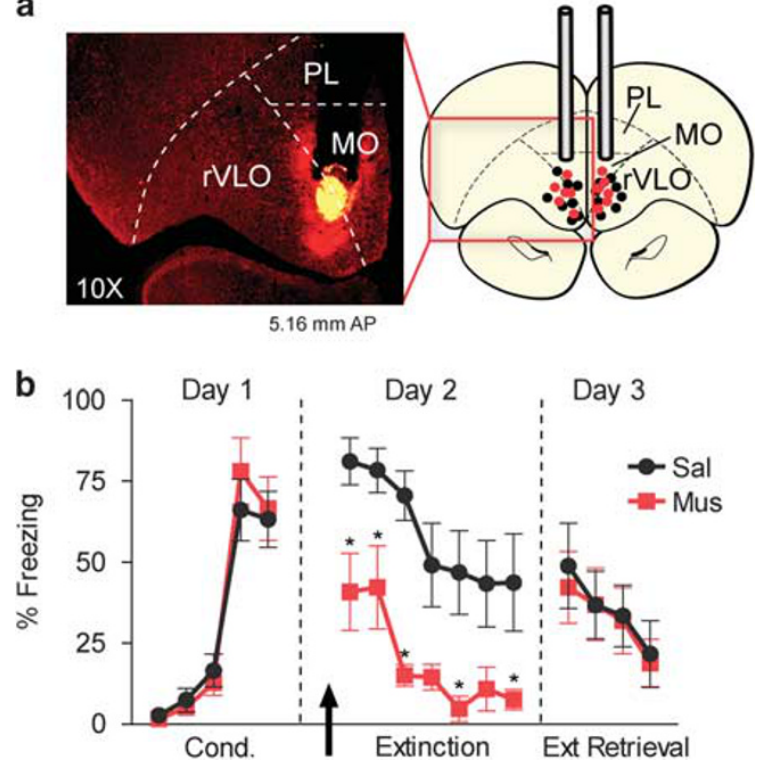

Figure 3 Inhibition of medial OFC (mOFC) neurons reduces fear expression. (a) Left: representative micrograph showing the spread of muscimol (MUS) in mOFC. Right: placement of cannula tips within the mOFC. (b) Freezing plots for saline (SAL, $n=8)$ and MUS $(n=6)$ groups. MUS reduced freezing on day 2 and did not alter extinction retrieval on day 3. Data are shown in blocks of two trials as mean \pm SEM. $* p<0.05$. a
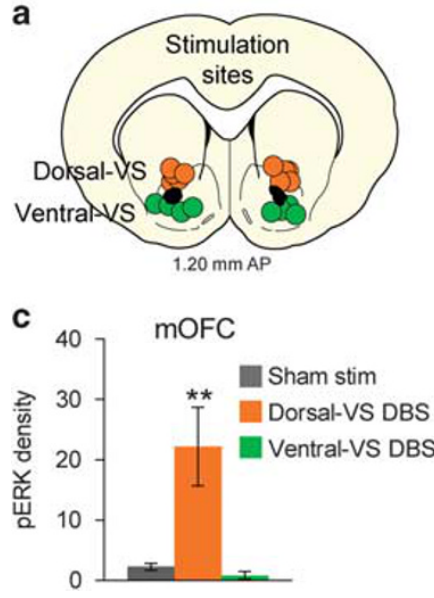
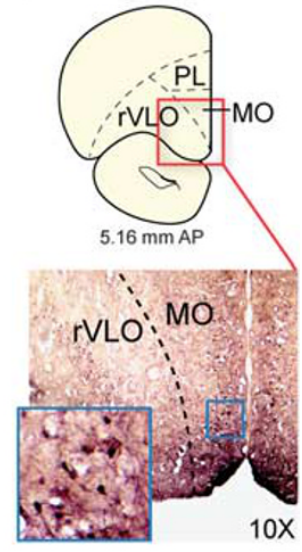

Figure 4 Deep brain stimulation (DBS) of dorsal-ventral striatum (VS) increases pERK expression in medial OFC (mOFC) neurons. (a) DBS electrode placements in ventral striatum. Circle diameter indicates the estimated spread of current from electrode tip. DBS of dorsal-VS was applied dorsal to the anterior commissure (orange circles) and DBS of ventral-VS was applied ventral to the anterior commissure (green circles). (b) Representative micrograph showing pERK labeling in mOFC after DBS of dorsal-VS. (c) Comparison of pERK density (counts $/ 0.1 \mathrm{~mm}^{2}$ ) after rats underwent $3 \mathrm{~h}$ of Sham DBS $(n=9)$, dorsal-VS DBS $(n=4)$ or ventral-VS DBS $(n=4)$. Data presented as mean \pm SEM. $* * * 0<0$.

involved. Deep layers of PL project to the lateral hypothalamus (Gabbott et al, 2005), which mediate conditioninginduced increases in autonomic responses (LeDoux et al, 1988). Another target of PL deep layers is the dorsal midline thalamus (Gabbott et al, 2005), an area shown to be important for fear expression (Padilla-Coreano et al, 2012; Do-Monte et al, 2015). Thus, the beneficial effects of DBS in dorsal-VS (rather than ventral-VS) may rely on mOFC and PL convergence at this site, which has been observed in rats, monkeys, and humans (Draganski et al, 2006; Haber et al, 2006; Mailly et al, 2013).

In addition to reducing freezing, DBS of dorsal-VS has also been shown to strengthen extinction memory (Rodriguez-Romaguera et al, 2012). Potentiation of inhibitory circuitry within OFC or PL by DBS may account for strengthening of extinction. Consistent with this, we observed expression of pERK in both mOFC (this study) and PL (Rodriguez-Romaguera et al, 2012) following DBS. As pharmacological inactivation of mOFC (this study) or PL (Sierra-Mercado et al, 2011) does not alter extinction memory, DBS-induced inhibition of these areas likely does not replace extinction. In support of this, administering DBS alone does not induce extinction (RodriguezRomaguera et al, 2012). Alternatively, DBS may strengthen extinction memory via indirect effects on IL, as suggested by DBS-induced increases in Fos, pERK, and BDNF levels in IL (Do-Monte et al, 2013; Rodriguez-Romaguera et al, 2012).

There is considerable debate over the homology of orbitofrontal areas in rodents and primates. Cross-species comparisons of connectivity suggest that rodent mOFC is homologous to the medial region of the OFC in humans (Brodmann area 14) (Price, 2007; Wallis, 2012; Wise, 2008). Hyperactivity within the human mOFC is thought to drive the excessive compulsive behavior that is the hallmark of OCD (for reviews, see Baxter et al, 1996; Menzies et al, 2008; Milad and Rauch, 2012). DBS of the VC/VS increases BOLD signaling in mOFC of OCD patients (Rauch et al, 2006); however, whether this represents activation of excitatory or inhibitory networks is not known. Furthermore, a recent study showed that optogenetic activation of mOFC neurons induces OCD-like behaviors in mice (Ahmari et al, 2013). Therefore, DBS-induced inhibition of mOFC may explain the reduction in symptoms observed in refractory OCD patients receiving DBS (Denys et al, 2010; Greenberg et al, 2010a; Haber and Behrens, 2014). In support of this, it was recently shown that DBS of VC/VS reduces functional connectivity in cortico-striatal circuits in OCD patients (Figee et al, 2013). Thus, augmentation of exposure-based therapies with DBS (Denys et al, 2010) may be due to enhancement of fear extinction via mOFC inhibition.

\section{FUNDING AND DISCLOSURE}

The authors declare no conflict of interest.

\section{ACKNOWLEDGEMENTS}

We thank the members of the Silvio O. Conte Center for Research in OCD: Barry W Connors, Darin Dougherty, Emad N. Eskandar, Anthony A Grace, Benjamin D Greenberg, Mohammed R Milad, and Steven A Rasmussen for useful discussions. We also thank Julia F Lehman, Anna E. Borkowska-Belanger, Carlos Rodriguez, and Zarkalys Quintero for technical assistance. This work was supported by NIH grants P50 MH086400 (to SNH and GJQ), R37 
MH058883 and R01 MH081975 (to GJQ), R36 MH105039 (to JRR), and the University of Puerto Rico President's Office.

\section{REFERENCES}

Ahmari SE, Spellman T, Douglass NL, Kheirbek MA, Simpson HB, Deisseroth K et al (2013). Repeated cortico-striatal stimulation generates persistent OCD-like behavior. Science 340: 1234-1239.

Baxter LR Jr., Saxena S, Brody AL, Ackermann RF, Colgan M, Schwartz JM et al (1996). Brain mediation of obsessivecompulsive disorder symptoms: evidence from functional brain imaging studies in the human and nonhuman primate. Semin Clin Neuropsychiatry 1: 32-47.

Berendse HW, Galis-de Graaf Y, Groenewegen HJ (1992). Topographical organization and relationship with ventral striatal compartments of prefrontal corticostriatal projections in the rat. J Comp Neurol 316: 314-347.

Burgos-Robles A, Vidal-Gonzalez I, Santini E, Quirk GJ (2007). Consolidation of fear extinction requires NMDA receptordependent bursting in the ventromedial prefrontal cortex. Neuron 53: 871-880.

Corcoran KA, Quirk GJ (2007). Activity in prelimbic cortex is necessary for the expression of learned, but not innate, fears. $J$ Neurosci 27: 840-844.

Denys D, Mantione M, Figee M, van den Munckhof P, Koerselman F, Westenberg $\mathrm{H}$ et al (2010). Deep brain stimulation of the nucleus accumbens for treatment-refractory obsessive-compulsive disorder. Arch Gen Psychiatry 67: 1061-1068.

Do-Monte FH, Rodriguez-Romaguera J, Rosas-Vidal LE, Quirk GJ (2013). Deep brain stimulation of the ventral striatum increases BDNF in the fear extinction circuit. Front Behav Neurosci 7: 102.

Do-Monte FH, Quinones-Laracuente K, Quirk GJ (2015). A temporal shift in the circuits mediating retrieval of fear memory. Nature (in press).

Draganski B, Gaser C, Kempermann G, Kuhn HG, Winkler J, Buchel C et al (2006). Temporal and spatial dynamics of brain structure changes during extensive learning. J Neurosci 26: 6314-6317.

Figee M, Luigjes J, Smolders R, Valencia-Alfonso CE, van Wingen G, de Kwaasteniet B et al (2013). Deep brain stimulation restores frontostriatal network activity in obsessive-compulsive disorder. Nat Neurosci 16: 386-387.

Gabbott PL, Warner TA, Jays PR, Salway P, Busby SJ (2005). Prefrontal cortex in the rat: projections to subcortical autonomic, motor, and limbic centers. J Comp Neurol 492: 145-177.

Gradinaru V, Mogri M, Thompson KR, Henderson JM, Deisseroth $\mathrm{K}$ (2009). Optical deconstruction of parkinsonian neural circuitry. Science 324: 354-359.

Greenberg BD, Gabriels LA, Malone DA Jr., Rezai AR, Friehs GM, Okun MS et al (2010a). Deep brain stimulation of the ventral internal capsule/ventral striatum for obsessive-compulsive disorder: worldwide experience. Mol Psychiatry 15: 64-79.

Greenberg BD, Rauch SL, Haber SN (2010b). Invasive circuitrybased neurotherapeutics: stereotactic ablation and deep brain stimulation for OCD. Neuropsychopharmacology 35: 317-336.

Haber SN, Behrens TE (2014). The neural network underlying incentive-based learning: implications for interpreting circuit disruptions in psychiatric disorders. Neuron 83: 1019-1039.

Haber SN, Kim KS, Mailly P, Calzavara R (2006). Reward-related cortical inputs define a large striatal region in primates that interface with associative cortical connections, providing a substrate for incentive-based learning. J Neurosci 26: 8368-8376.

Hoover WB, Vertes RP (2007). Anatomical analysis of afferent projections to the medial prefrontal cortex in the rat. Brain Struct Funct 212: 149-179.
Hoover WB, Vertes RP (2011). Projections of the medial orbital and ventral orbital cortex in the rat. J Comp Neurol 519: 3766-3801.

Kim SC, Jo YS, Kim IH, Kim H, Choi JS (2010). Lack of medial prefrontal cortex activation underlies the immediate extinction deficit. J Neurosci 30: 832-837.

Laurent V, Westbrook RF (2009). Inactivation of the infralimbic but not the prelimbic cortex impairs consolidation and retrieval of fear extinction. Learn Mem 16: 520-529.

LeDoux JE, Iwata J, Cicchetti P, Reis DJ (1988). Different projections of the central amygdaloid nucleus mediate autonomic and behavioral correlates of conditioned fear. J Neurosci 8: 2517-2529.

Mailly P, Aliane V, Groenewegen HJ, Haber SN, Deniau JM (2013). The rat prefrontostriatal system analyzed in 3D: evidence for multiple interacting functional units. J Neurosci 33: 5718-5727.

Maroun M, Kavushansky A, Holmes A, Wellman C, Motanis H (2012). Enhanced extinction of aversive memories by highfrequency stimulation of the rat infralimbic cortex. PLoS One 7: e35853.

McCracken CB, Grace AA (2007). High-frequency deep brain stimulation of the nucleus accumbens region suppresses neuronal activity and selectively modulates afferent drive in rat orbitofrontal cortex in vivo. J Neurosci 27: 12601-12610.

McGeorge AJ, Faull RL (1989). The organization of the projection from the cerebral cortex to the striatum in the rat. Neuroscience 29: 503-537.

Menzies L, Chamberlain SR, Laird AR, Thelen SM, Sahakian BJ, Bullmore ET (2008). Integrating evidence from neuroimaging and neuropsychological studies of obsessive-compulsive disorder: the orbitofronto-striatal model revisited. Neurosci Biobehav Rev 32: 525-549.

Milad MR, Furtak SC, Greenberg JL, Keshaviah A, Im JJ, Falkenstein MJ et al (2013). Deficits in conditioned fear extinction in obsessive-compulsive disorder and neurobiological changes in the fear circuit. JAMA Psychiatry 70: 608-618; quiz 554 .

Milad MR, Quirk GJ (2002). Neurons in medial prefrontal cortex signal memory for fear extinction. Nature 420: 70-74.

Milad MR, Rauch SL (2012). Obsessive-compulsive disorder: beyond segregated cortico-striatal pathways. Trends Cogn Sci 16: 43-51.

Milad MR, Vidal-Gonzalez I, Quirk GJ (2004). Electrical stimulation of medial prefrontal cortex reduces conditioned fear in a temporally specific manner. Behav Neurosci 118: 389-394.

Padilla-Coreano N, Do-Monte FH, Quirk GJ (2012). A timedependent role of midline thalamic nuclei in the retrieval of fear memory. Neuropharmacology 62: 457-463.

Paxinos G, Watson C (1998). The Rat Brain in Stereotaxic Coordinates. Academic Press: San Diego, Vol 4th.

Price JL (2007). Definition of the orbital cortex in relation to specific connections with limbic and visceral structures and other cortical regions. Ann NY Acad Sci 1121: 54-71.

Quirk GJ, Russo GK, Barron JL, Lebron K (2000). The role of ventromedial prefrontal cortex in the recovery of extinguished fear. J Neurosci 20: 6225-6231.

Rauch SL, Dougherty DD, Malone D, Rezai A, Friehs G, Fischman AJ et al (2006). A functional neuroimaging investigation of deep brain stimulation in patients with obsessivecompulsive disorder. J Neurosurg 104: 558-565.

Rodriguez-Romaguera J, Do Monte FH, Quirk GJ (2012). Deep brain stimulation of the ventral striatum enhances extinction of conditioned fear. Proc Natl Acad Sci USA 109: 8764-8769.

Schilman EA, Uylings HB, Galis-de Graaf Y, Joel D, Groenewegen HJ (2008). The orbital cortex in rats topographically projects to central parts of the caudate-putamen complex. Neurosci Lett 432: $40-45$. 
Sesack SR, Deutch AY, Roth RH, Bunney BS (1989). Topographical organization of the efferent projections of the medial prefrontal cortex in the rat: an anterograde tract-tracing study with Phaseolus vulgaris leucoagglutinin. J Comp Neurol 290: 213-242.

Sierra-Mercado D, Padilla-Coreano N, Quirk GJ (2011). Dissociable roles of prelimbic and infralimbic cortices, ventral hippocampus, and basolateral amygdala in the expression and extinction of conditioned fear. Neuropsychopharmacology 36: 529-538.

St Onge JR, Stopper CM, Zahm DS, Floresco SB (2012). Separate prefrontal-subcortical circuits mediate different components of risk-based decision making. J Neurosci 32: 2886-2899.
Sweatt JD (2004). Mitogen-activated protein kinases in synaptic plasticity and memory. Curr Opin Neurobiol 14: 311-317.

Vertes RP (2004). Differential projections of the infralimbic and prelimbic cortex in the rat. Synapse 51: 32-58.

Vidal-Gonzalez I, Vidal-Gonzalez B, Rauch SL, Quirk GJ (2006). Microstimulation reveals opposing influences of prelimbic and infralimbic cortex on the expression of conditioned fear. Learn Mem 13: 728-733.

Wallis JD (2012). Cross-species studies of orbitofrontal cortex and value-based decision-making. Nat Neurosci 15: 13-19.

Wise SP (2008). Forward frontal fields: phylogeny and fundamental function. Trends Neurosci 31: 599-608. 